\title{
Novel mutations in the IRF6 gene in Brazilian families with Van der Woude syndrome
}

\author{
LÍVIA MÁRIS RIBEIRO PARANAÍBA ${ }^{1}$, HERCÍLIO MARTELLI-JÚNIOR ${ }^{2,3}$, \\ MÁRIO SERGIO OLIVEIRA SWERTS ${ }^{3}$, SERGIO R.P. LINE ${ }^{4}$ and RICARDO D. COLETTA ${ }^{1}$
}

\begin{abstract}
${ }^{1}$ Department of Oral Diagnosis, School of Dentistry, State University of Campinas, Av. Limeira 901, CEP 13414-018
Piracicaba, São Paulo; ${ }^{2}$ Stomatology Clinic, Dental School, State University of Montes Claros, Caixa Postal 126, CEP 39401-089 Montes Claros, Minas Gerais; ${ }^{3}$ Center for Rehabilitation of Craniofacial Anomalies, Dental School, University of Alfenas, Rod. MG 179, Km 0, CEP 37130-000 Alfenas, Minas Gerais; ${ }^{4}$ Department of Morphology, School of Dentistry, State University of Campinas, Av. Limeira 901, CEP 13414-018 Piracicaba, São Paulo, Brazil
\end{abstract}

Received April 17, 2008; Accepted July 3, 2008

DOI: 10.3892/ijmm_00000049

\begin{abstract}
Van der Woude Syndrome (VWS) is an autosomal craniofacial disorder characterized by lower lip pits and cleft lip and/or palate. Mutations in the interferon regulatory factor 6 (IRF6) gene have been identified in patients with VWS. To identify novel IRF6 mutations in patients affected by VWS, we screened 2 Brazilian families, sequencing the entire IRF6coding region and flanking intronic boundaries. Two novel heterozygous mutations were identified: a frame shift mutation with deletion of $\mathrm{G}$ at the nucleotide position 520 in the exon 6 (520delG), and a missense single nucleotide substitution from $\mathrm{T}$ to $\mathrm{A}$ at nucleotide position 1135 in exon 8 (T1135A). By using restriction enzyme analysis, we were able to demonstrate the lack of similar mutations in unrelated healthy individuals and non-syndromic cleft lip and palate patients. Our results further confirmed that haploinsufficiency of the IRF6 gene results in VWS.
\end{abstract}

\section{Introduction}

Van der Woude syndrome (VWS) (OMIM \#119300) is a rare craniofacial disorder characterized by lower lip pits, cleft lip and/or palate $(\mathrm{CL} / \mathrm{P})$ and, occasionally, hypodontia $(1,2)$. This syndrome occurs in approximately $2 \%$ of CL/P patients, and is distinguished from non-syndromic CL/P by the presence of lip pits (3). Its incidence is estimated at 1-2.5 cases per 100,000 live births (4). VWS is transmitted in an autosomal dominant mode with high penetrance and varied clinical expression (expressivity); however, $30-50 \%$ of VWS cases

Correspondence to: Dr Ricardo D. Coletta, Department of Oral Diagnosis, School of Dentistry, State University of Campinas, Av. Limeira 901, CEP 13414-018 Piracicaba, São Paulo, Brazil

E-mail: coletta@fop.unicamp.br

Key words: Van der Woude syndrome, interferon regulatory factor 6 gene, mutation represent de novo mutations $(1,4,5)$. Mutations in the interferon regulatory factor 6 (IRF6) gene have been found in patients with VWS (6). To date, more than 70 different mutations in the IRF6 gene have been reported in VWS affected patients worldwide (5-17). IRF6 belongs to a family of 9 transcription factors that share a highly conserved helix DNA-binding, and a protein-interactive domain in the carboxyl-terminal region called Smad-interferon regulatory factor-binding domain (SMIR) (18). Most IRF members regulate the expression of interferon $\alpha$ and $\beta$ after viral infection (19), but the exact function of IRF6 remains unknown. Mutations in the IRF6 gene also can cause popliteal pterygium syndrome (MIM \#119500), which shares some common features with VWS (14).

We recently described the clinical features of 2 large Brazilian families affected by VWS (20). In the first family, VWS was transmitted as an autosomal dominant character with apparent low penetrance. All the affected members demonstrated association of bilateral paramedian lower lip pits and CL/P. In the second family, segregation of VWS was consistent with an autosomal dominant mode with penetrance appearing complete, and the expressivity was distinct among affected members. Here, by sequencing the entire coding region of the IRF6 gene of the 2 VWS families, we located 2 novel mutations (520delG and T1135A). The T1135A mutation is located in the SMIR protein-interactive domain and the 520delG abolishes this region, presumably affecting the regulatory functions of IRF6 in the transcriptional control of the downstream target genes.

\section{Materials and methods}

Families with VWS and controls. Pedigree and clinical details of the 2 VWS families were previously described (20). From Family 1, which is composed of 54 descendants with 12 presented features of VWS, sequencing analysis was performed on 6 affected members and 10 unaffected members. In Family 2, comprising 19 descendants with 8 affected by VWS, sequencing analysis was performed on all members.

As a control, 100 unrelated healthy individuals and 50 patients with non-syndromic CL/P were recruited and 

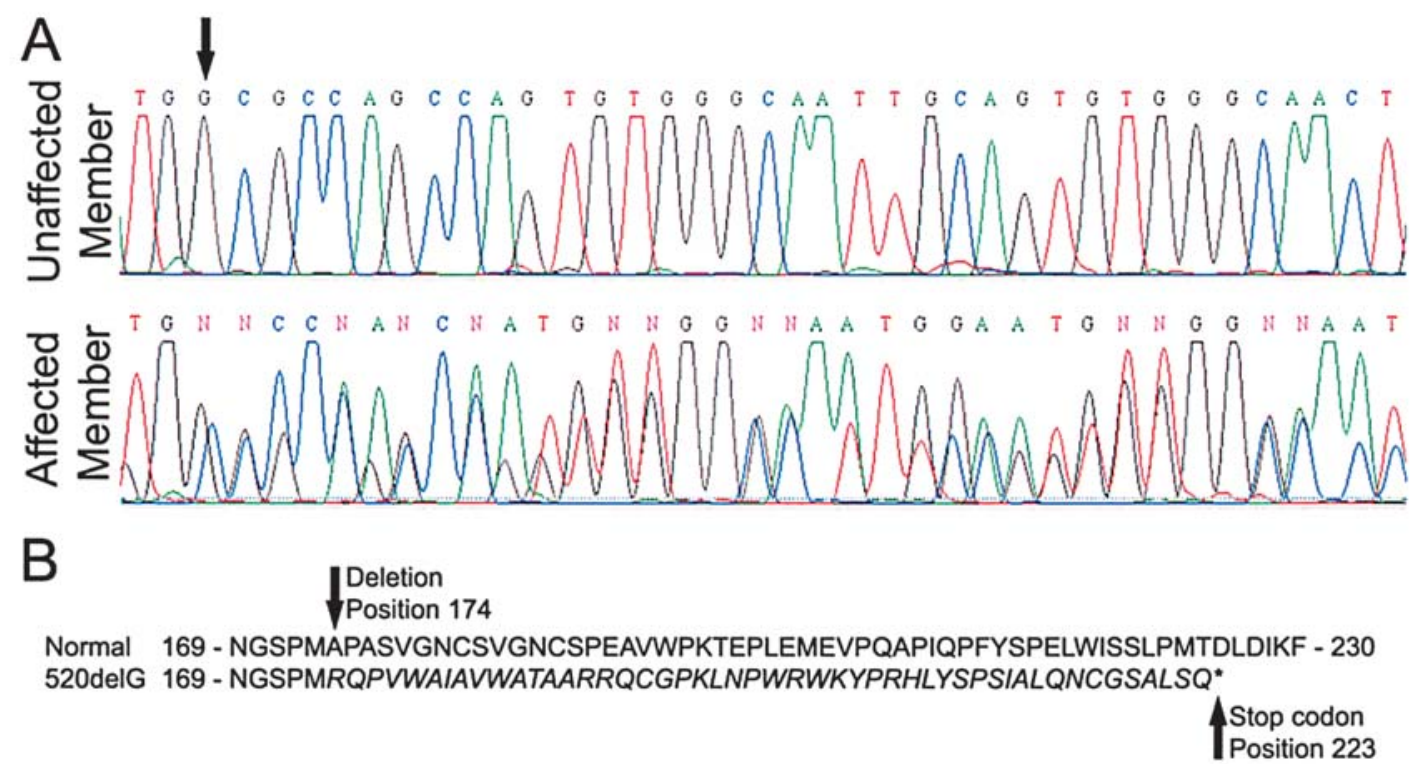

Figure 1. Identification of an undescribed IRF6 mutation in the VWS Family 1 of this study. (A) Portions of representative DNA-sequence eletropherograms of an unaffected (top) and an affected (bottom) member of Family 1. Arrow indicates the altered nucleotide. The affected member is heterozygous for the deletion of $\mathrm{G}$ at the nucleotide position 520 of IRF6 (520delG). (B) A portion of the normal amino acid sequence of IRF6 was aligned with the mutated sequence. The amino acid sequence newly created by the $520 \mathrm{delG}$ mutation is indicated in italics. Note that this mutation resulted in the subsequent change of 49 amino acids, ending in a premature termination at amino acid 223.

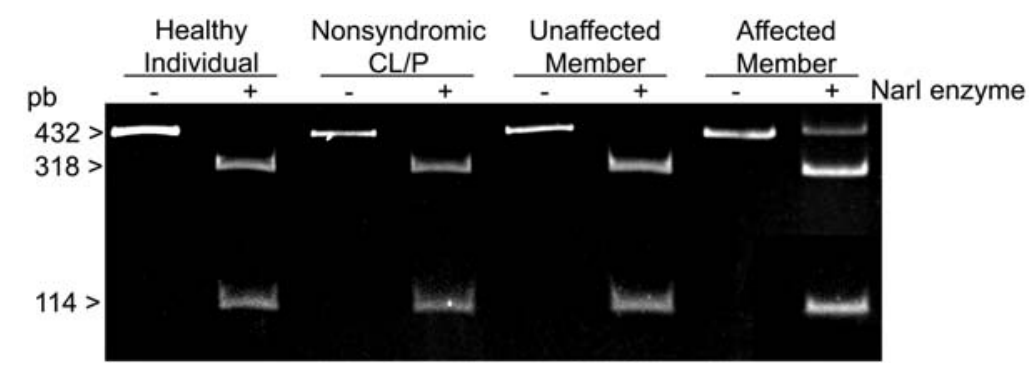

Figure 2. Restriction enzyme analysis of the 520delG mutation. Representative samples from 1 healthy individual, 1 non-syndromic CL/P patient, and 2 members of Family 1 ( 1 unaffected and 1 affected member). The 520delG mutation eliminates the NarI site, thus preserving the digestion of the 432 fulllength amplicons generated from the mutated allele. PCR-amplified DNA from VWS individuals showed the 432-bp fragment in addition to the 318- and 114-bp fragments originating from the normal digested allele. DNA from healthy individuals, non-syndromic CL/P patients, and unaffected family members demonstrated only the 318- and 114-bp fragments.

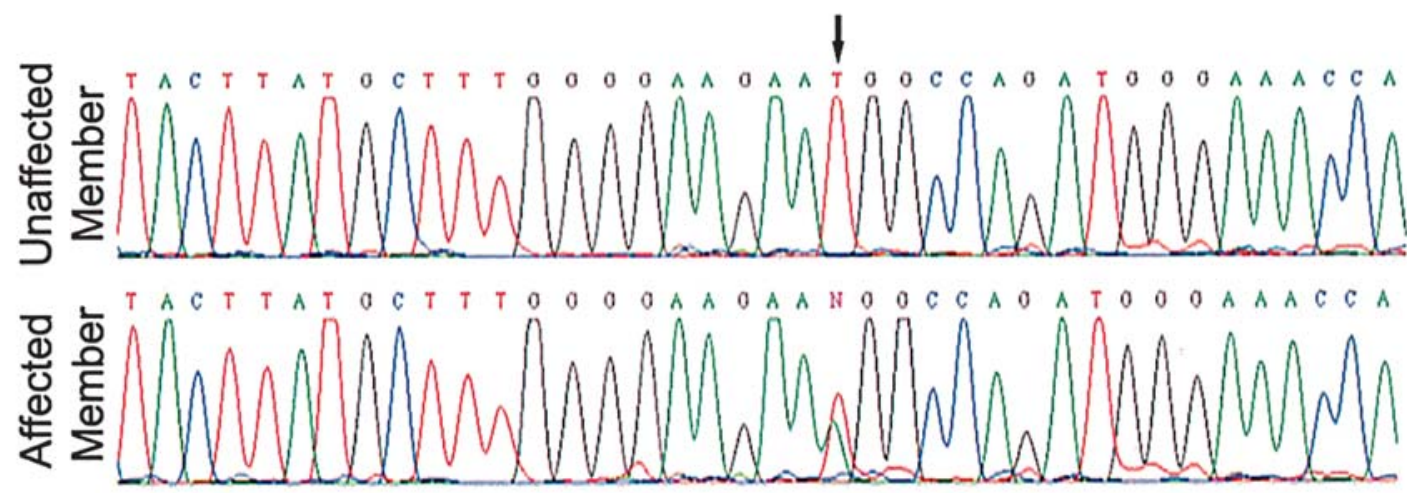

Figure 3. DNA-sequence eletropherograms showing the IRF6 mutation identified in VWS patients of Family 2. The top eletropherogram represents a portion of IRF6 exon 8 of an unaffected member. The results of an affected member are demonstrated on the bottom eletropherogram. VWS patients are heterozygous at position 1135, as revealed by the transversion of T to A (arrow), causing the substitution of a tryptofano to arginine (W379R). 


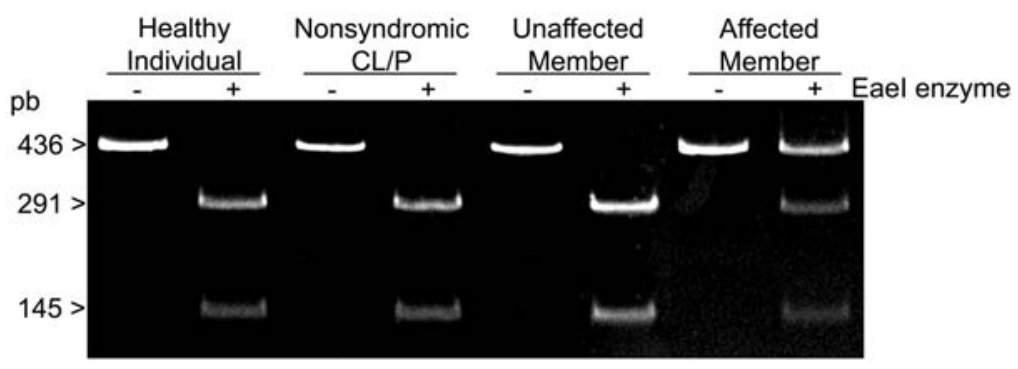

Figure 4. Restriction enzyme analysis of the T1135A mutation. Representative samples from 1 healthy individual, 1 non-syndromic CL/P patient, and 2 members of Family 2 (1 unaffected and 1 affected member), digested with EaeI. Digestion of the PCR product from affected family members resulted in 3 fragments: 1 undigested fragment, 436 bp and 2 digested fragments, 291 and 145 bp, confirming the heterozygous T1135A mutation.

examined. Written informed consent was obtained from all participants or their guardians before the study commenced. This study was carried out with the approval of the Human Research Ethics Committee of the University.

Sequence analysis. Genomic DNA was extracted from oral mucosa cells as previously described (21). Exons 1-9 and their flanking splice junctions were PCR amplified with specific primers (9). This was followed by direct sequencing in an ABI PRISM 3100 Genetic Analyzer (Applied Biosystems, Hitachi). Detected mutations in affected members were confirmed by repetition of the sequence analysis on new DNA samples, and by restriction enzyme analysis.

Restriction enzyme analysis. Restriction enzyme analysis was performed to confirm the mutations in affected members and to analyze the control individuals. Exon 6 PCR products amplified from Family 1 and control DNA samples, were directly incubated with the NarI restriction enzyme (New England Biolabs Inc., Ipswich, MA, USA), whereas exon 8 PCR products from Family 2 and controls were incubated with the EaeI restriction enzyme (Fermentas Inc., Hanover, MD, USA). The same pairs of primers for the sequencing analysis were used here. After incubation, the products were electrophoresed on an $8 \%$ non-denaturing polyacrylamide gel containing $0.5 \mu \mathrm{g} / \mathrm{ml}$ of ethidium bromide.

\section{Results}

All affected members of Family 1 showed a heterozygous G deletion at nucleotide position 520 of IRF6 cDNA (520delG), the first nucleotide of alanine positioned at 174 (Ala174) of the amino acid sequence of IRF6 protein (Fig. 1). This mutation resulted in the subsequent change of 49 amino acids and a premature termination at amino acid 223, abolishing the carboxyl-terminal region of IRF6. The truncated protein caused by this frame shift mutation is expected to lack the SMIR domain, presumably important for interacting with Smad transcription factors. Two of the 10 unaffected members of this family demonstrated the $\mathrm{G}$ deletion, and were being considered carrier members. To further confirm this finding, we performed restriction enzyme analysis in all Family 1 members and in the control individuals (Fig. 2). The 520delG mutation eliminates the NarI site, thus preserving the digestion of 432 full-length amplicons generated from the mutated allele. PCR-amplified DNA from VWS individuals showed the 432-bp fragment in addition to the 318- and 114-bp fragments from the normal digested allele, while unaffected family members, healthy control individuals and non-syndromic CL/P patients demonstrated only the 318- and 114-bp fragments (Fig. 2).

In Family 2, the heterozygous $\mathrm{T}$ to $\mathrm{A}$ transversion at nucleotide position 1135 of exon 8 of IRF6 (T1135A) resulted in a tryptofano to arginine substitution at codon 379 (W379R) of the IRF6 amino acid sequence (Fig. 3). The 379 residue is located in the SMIR-binding domain, which is absolutely conserved in the IRF6 orthologues. One clinically unaffected member of Family 2 demonstrated this mutation. Since the T to A transversion abolishes the EaeI restriction site in the exon 8 , we confirmed the missense mutation (1135 T>A) by incubating PCR products directly with EaeI enzyme. Exon 8 PCR products from unaffected family members, healthy individuals and non-syndromic CL/P, incubated with EaeI resulted in 2 fragments (145 and $291 \mathrm{bp}$ ). However, enzyme incubation with PCR products from affected family members resulted in 3 fragments [1 undigested, originating from the mutated amplicon (436 bp) and 2 digested fragments], confirming the specificity of the mutation (Fig. 4).

\section{Discussion}

Since the first identification of the IRF6 mutation in VWS patients by Kondo et al (6), more than 70 different mutations have been reported in patients with this syndrome. The vast majority are missense mutations, products of single amino acid substitution, located in the DNA-binding domain or in the protein-interactive domain of IRF6 $(8,11,13,22)$. Mutations in the regions presumably affected the regulatory function of the IRF6 protein at the transcriptional control of the downstream target genes (23). The mutation detected in Family 1 (520delG) was not located in the DNA-binding domain or in the SMIR domain of the IRF6 gene. However, the nucleotide deletion caused an alteration in the codon-reading, introducing a premature termination at exon 7 level and resulting in a protein lacking the entire SMIR domain. Although the precise function of IRF6 is unclear, the lack of the SMIR domain inhibits the interaction with Smad transcription factors, which are well known to transduce transforming growth factor- $\beta$ signals, that play an essential role in palate and lip formation 
and fusion $(24,25)$. In IRF3 and IRF7 orthologues, Smad binding to the SMIR domain is required for interaction with other transcription factors in the regulation of target genes (26). It is presumed that the SMIR domain also plays an essential role in the dimerization of IRF6 protein (26).

The tryptophane to arginine substitution (W379R) observed in the affected members of Family 2, occurred in the SMIR domain. This residue of tryptophane at position 379 is conserved in all 9 members of the IRF family. Around this amino acid, other substitutions, including phenylalanine to serine at position 369 (F369S), cysteine to tyrosine at position 374 (C374Y), and lysine to glutamic acid at position 388 (K388E) have been reported $(6,11,16)$. Notably, at this same tryptophane residue, Wang et al (8) related a TGG to TGA transition, resulting in a premature stop codon (p.W379X). By using bioinformatics analysis $(27,28)$, we observed that the tryptophane to arginine substitution resulted in an alteration in the hydrophobic profile of the protein and in a shift in the protein secondary structure, suggesting biological effects in the regulatory activity of IRF6. Collectively, our data demonstrate that mutations in IRF6, resulting in alterations in the SMIR domain, are etiological for VWS, since those same mutations were not found in 100 healthy control individuals and in 50 control patients with isolated CL/P.

VWS is characterized by high penetrance and varied expressivity $(1,4,5)$. Pedigree analysis of Family 1 suggested a low penetrance (20), which was confirmed by mutation analysis. Two clinically unaffected members showed the same mutation observed in the VWS members. Notably, one of the carriers had an affected twin brother, and the second carrier had her mother and one of her 5 siblings phenotypically and genotypically affected. In Family 2, one member with no signs of VWS was a mutation carrier. This implies incomplete penetrance of the T1135A mutation, contrasting with the clinical analysis of the pedigree. Although some studies state that low penetrance is due to the lack of recognition of patients with microforms or subclinical forms of the disease (29), in this study, all patients were carefully examined. Comparing the 2 mutations, the 520delG mutation resulted in a truncated protein and in a homogeneous and more severe phenotype. The lack of the entire SMIR domain probably abolishes the protein function. However, single nucleotide substitution in the SMIR domain, resulting in normal-sized protein, under the influence of stochastic factors or modifier genes, produced a protein with imprecise functions, resulting in a marked phenotypic variation. Mechanisms underlying penetrance and expressivity include the influence of alleles, modifier genes, gender, and environmental factors (30), so VWS is subject to genetic and environmental variation. It is becoming clear that there are many allelic variants throughout the genome, and these are not just coding variants. Differences in transcriptional control through promoter and enhancer differences are increasingly emerging $(31,32)$. Furthermore, gene expression is clearly subject to complex epigenetic control (33), and we are just beginning to understand the rules involved in the modulation of chromatin structure (34). Finally, environmental variation plays a major role in modulating all aspects of gene expression, particularly in CLP (35), but individual responses to environmental factors are under partial genetic control. Each mutation observed in any individual is unique in its physiological, genomic, environmental and spatiotemporal context.

In conclusion, we reported 2 novel mutations (520delG and T1135A) in Brazilian families with VWS. These mutations produced an impaired SMIR domain, suggesting that this domain is essential for IRF6 function. Furthermore, VWS occurs from the haploinsufficiency of IRF6, suggesting a direct participation of IRF6 in craniofacial development. High levels of IRF6 mRNA were identified along the medial edge of the fusing palate during embryogenesis of mice (6).

\section{Acknowledgements}

We are grateful to Drs Letízia Monteiro de Barros and Marcelo Reis Chaves for assistance with the sample collection. We also thank Dr Siu Mui Tsai for allowing us to use a DNA sequencer. This work was supported by grants from The Minas Gerais State Research Foundation-FAPEMIG, Brazil, and from The National Council for Scientific and Technological Development-CNPq, Brazil. L.M.R Paranaíba is supported by Fundação Coordenação de Aperfeiçoamento de Pessoal de Nível Superior-CAPES, Brazil.

\section{References}

1. Huang JJ, Hou JW, Tan YC, Chen KT, Lo LJ and Chen YR: Van der Woude syndrome: clinical presentation in 64 patients. Cleft Palate Craniofac J 44: 649-652, 2007.

2. Oberoi S and Vargervik K: Hypoplasia and hypodontia in Van der Woude syndrome. Cleft Palate Craniofac J 42: 459-466, 2005.

3. Janku P, Robinow M, Kelly T, Bralley R, Baynes A and Edgerton MT: The van der Woude syndrome in a large kindred: variability, penetrance, genetic risks. Am J Med Genet 5: 117-123, 1980.

4. Rizos M and Spyropoulos MN: Van der Woude syndrome: a review. Cardinal signs, epidemiology, associated features, differential diagnosis, expressivity, genetic counselling and treatment. Eur J Orthod 26: 17-24, 2004.

5. Item CB, Turhani D, Thurnher D, et al: Van Der Woude syndrome: variable penetrance of a novel mutation (p.Arg 84Gly) of the IRF6 gene in a Turkish family. Int J Mol Med 15: 247-251, 2005.

6. Kondo S, Schutte BC, Richardson RJ, et al: Mutations in IRF6 cause Van der Woude and popliteal pterygium syndromes. Nat Genet 32: 285-289, 2002.

7. Brosch S, Baur M, Blin N, Reinert S and Pfister M: A novel IRF6 nonsense mutation (Y67X) in a German family with Van der Woude syndrome. Int J Mol Med 20: 85-89, 2007.

8. Wang X, Liu J, Zhang H, et al: Novel mutations in the IRF6 gene for Van der Woude syndrome. J Hum Genet 113: 382-386, 2003.

9. Kayano S, Kure S, Suzuki Y, et al: Novel IRF6 mutations in Japanese patients with Van der Woude syndrome: two missense mutations (R45Q and P396S) and a 17-kb deletion. J Hum Genet 48: 622-628, 2003

10. Kim Y, Park JY, Lee TJ and Yoo HW: Identification of two novel mutations of IRF6 in Korean families affected with Van der Woude syndrome. Int J Mol Med 12: 465-468, 2003.

11. Peyrard-Janvid M, Pegelow M, Koillinen H, et al: Novel and de novo mutations of the IRF6 gene detected in patients with Van der Woude or popliteal pterygium syndrome. Eur J Hum Genet 13: 1261-1267, 2005.

12. Gatta V, Scarciolla O, Cupaioli M, Palka C, Chiesa PL and Stuppia L: A novel mutation of the IRF6 gene in an Italian family with Van der Woude syndrome. Mutat Res 547: 49-53, 2004.

13. Ye XQ, Jin HX, Shi LS, et al: Identification of novel mutations of IRF6 gene in Chinese families with Van der Woude syndrome. Int J Mol Med 16: 851-856, 2005.

14. Ghassibe M, Revencu N, Bayet B, et al: Six families with van der Woude and/or popliteal pterygium syndrome: all with a mutation in the IRF6 gene. J Med Genet 41: e15, 2004. 
15. Matsuzawa N, Yoshiura K, Machida J, et al: Two missense mutations in the IRF6 gene in two Japanese families with Van der Woude syndrome. Oral Surg Oral Med Oral Pathol Oral Radiol Endod 98: 414-417, 2004.

16. Matsuzawa N, Shimozato K, Natsume N, Niikawa N and Yoshiura K: A novel missense mutation in Van der Woude syndrome: usefulness of fingernail DNA for genetic analysis. J Dent Res 85: 1143-1146, 2006.

17. Du X, Tang W, Tian W, et al: Novel IRF6 mutations in Chinese patients with Van der Woude syndrome. J Dent Res 85: 937-940, 2006.

18. Takaoka $\mathrm{A}$, Tamura $\mathrm{T}$ and Taniguchi $\mathrm{T}$ : Interferon regulatory factor family of transcription factors and regulation of oncogenesis. Cancer Sci 99: 467-478, 2008.

19. Ozato K, Tailor $\mathrm{P}$ and Kubota T: The interferon regulatory factor family in host defense: mechanism of action. J Biol Chem 282: 20065-20069, 2007.

20. Martelli-Junior H, Chaves MR, Swerts MS, de Miranda RT, Bonan PR and Coletta RD: Clinical and genetic features of Van der Woude syndrome in two large families in Brazil. Cleft Palate Craniofac J 44: 239-243, 2007.

21. Aidar M and Line SR: A simple and cost-effective protocol for DNA isolation from buccal epithelial cells. Braz Dent J 18: 148-152, 2007.

22. Dissemond J, Haberer D, Franckson T and Hillen U: The Van der Woude syndrome: a case report and review of the literature. J Eur Acad Dermatol Venereol 18: 611-613, 2004.

23. Ingraham $\mathrm{CR}$, Kinoshita $\mathrm{A}$, Kondo $\mathrm{S}$, et al: Abnormal skin, limb and craniofacial morphogenesis in mice deficient for interferon regulatory factor 6 (Irf6). Nat Genet 38: 1335-1340, 2006.

24. Knight AS, Schutte BC, Jiang R and Dixon MJ: Developmental expression analysis of the mouse and chick orthologues of IRF6: the gene mutated in Van der Woude syndrome. Dev Dyn 235: 1441-1447, 2006.
25. Richardson RJ, Dixon J, Malhotra S, et al: Irf6 is a key determinant of the keratinocyte proliferation-differentiation switch. Nat Genet 38: 1329-1334, 2006

26. Au WC, Yeow WS and Pitha PM: Analysis of functional domains of interferon regulatory factor 7 and its association with IRF-3. Virology 280: 273-282, 2001.

27. Hall TA: BioEdit: a user-friendly biological sequence alignment editor and analysis program for Windows 95/98/NT. Nucleic Acids Symp Ser 41: 95-98, 1999.

28. McGuffin LJ, Bryson K and Jones DT: The PSIPRED protein structure prediction server. Bioinformatics 16: 404-405, 2000.

29. Wienker TF, Hudek G, Bissbort S, Mayerova A, Mauff G and Bender K: Linkage studies in a pedigree with Van der Woude syndrome. J Med Genet 24: 160-162, 1987.

30. Van Heyningen V and Yeyati PL: Mechanisms of nonMendelian inheritance in genetic disease. Hum Mol Genet 13: R225-R233, 2004.

31. Pastinen T, Sladek R, Gurd S, et al: A survey of genetic and epigenetic variation affecting human gene expression. Physiol Genomics 16: 184-193, 2004.

32. Yan H, Yuan W, Velculescu VE, Vogelstein B and Kinzler KW: Allelic variation in human gene expression. Science 297: 1143, 2002.

33. Wolffe AP and Matzke MA: Epigenetics: regulation through repression. Science 286: 481-486, 1999.

34. Dellino GI, Schwartz YB, Farkas G, McCabe D, Elgin SC and Pirrotta V: Polycomb silencing blocks transcription initiation. Mol Cell 13: 887-893, 2004.

35. Wong FK and Hagg U: An update on the aetiology of orofacial clefts. Hong Kong Med J 10: 331-336, 2004. 